\title{
A surgically treated arteriovenous fistula between the vertebral artery and internal jugular vein after insertion of a central venous catheter for mitral valve replacement
}

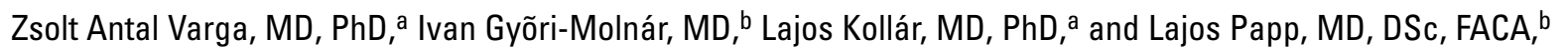
Pécs, Hungary

From the Department of Surgery and Vascular Surgery, ${ }^{\mathrm{a}}$ Baranya County Hospital, University of Pécs, Faculty of Medicine, and the Heart Institute and University of Pécs, ${ }^{b}$ Faculty of Medicine, Pécs, Hungary.

Received for publication May 29, 2001; accepted for publication July 24, 2001.

Address for reprints: Zsolt Antal Varga, MD, PhD, Department of Surgery and Vascular Surgery, Baranya County Hospital, University of Pécs, Faculty of Medicine, 2 Rákóczi u. Pécs 7623, Hungary (E-mail: zsolt55@ @otmail.com).

J Thorac Cardiovasc Surg 2002;123:575-7

Copyright $(\odot) 2002$ by The American Association for Thoracic Surgery

$0022-5223 / 2002 \$ 35.00+0 \quad \mathbf{1 2 / 5 4 / 1 1 9 8 7 8}$

doi:10.1067/mtc.2002.119878 case of iatrogenic arteriovenous (AV) fistula with mild
symptoms is presented.

Clinical Summary

A 57-year-old woman was seen for mild dyspnea and a locomotive murmur, which was heard by the patient in the horizontal position in her right ear right after her cardiac operation. Mitral valve replacement with myocardial pacemaker implantation and aortic valvuloplasty had been performed for grade III-IV mitral valve insufficiency 6 weeks before her presentation. For that operation, central venous monitoring was achieved by right internal jugular vein puncture. No complications developed during anesthesia or 


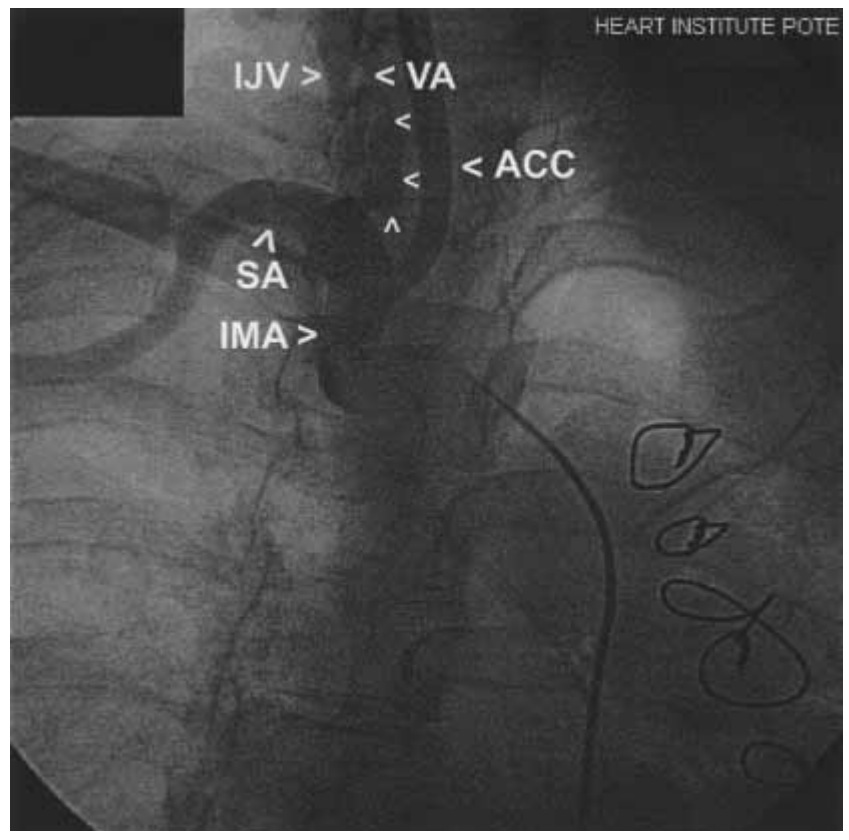

Figure 1. Selective innominate artery angiogram (right anterior oblique $33^{\circ}$ and caudal $2^{\circ}$ ) showing the AV fistula and the early filling of the internal jugular vein. IJV, Internal jugular vein; $S A$, subclavian artery; $A C C$, common carotid artery; VA, vertebral artery; IMA, internal thoracic artery. The AV fistula is marked by arrowheads.

surgery. At examination, a 2-mm puncture site scar was seen at the typical site and a locomotive murmur was heard and could be palpated on the right side of the neck. Dilation of the superficial venous network was not seen. A duplex sonogram showed an arterial jet in the internal jugular vein, but the exact site of the AV communication could not be visualized. The patient was hospitalized, warfarin sodium suspended, and subcutaneous low-molecularweight heparin anticoagulation was instituted. A selective angiogram of the innominate artery was performed via right femoral arterial puncture. Early venous filling was observed in the internal jugular vein from the shunt originating at the takeoff of the vertebral artery (Figure 1). After informed consent had been obtained from the patient, surgery was undertaken.

The second portion of the right subclavian artery was exposed via a supraclavicular incision. After proximal and distal control of the subclavian artery had been obtained, the vertebral artery, internal thoracic artery, and internal jugular vein were isolated. At the takeoff of the vertebral artery, a localized, mild inflammation in the surrounding connective tissue was found and a $3-\mathrm{cm}$ long AV fistula was isolated (Figure 2). After intravenous administration of 10,000 IU sodium heparin, the fistula was temporally clamped but the heart rate did not slow on the electrocardiographic monitor (no Nicoladoni-Branham sign). A venous blood sample, drawn from the internal jugular vein $1 \mathrm{~cm}$ cephalad to the venous entry site of the shunt before and after clamping of the fistula, showed higher oxygen saturation $(75,1 \%)$ before the fistula was clamped and lower $(63,5 \%)$ after the fistula was clamped. The venous site of the

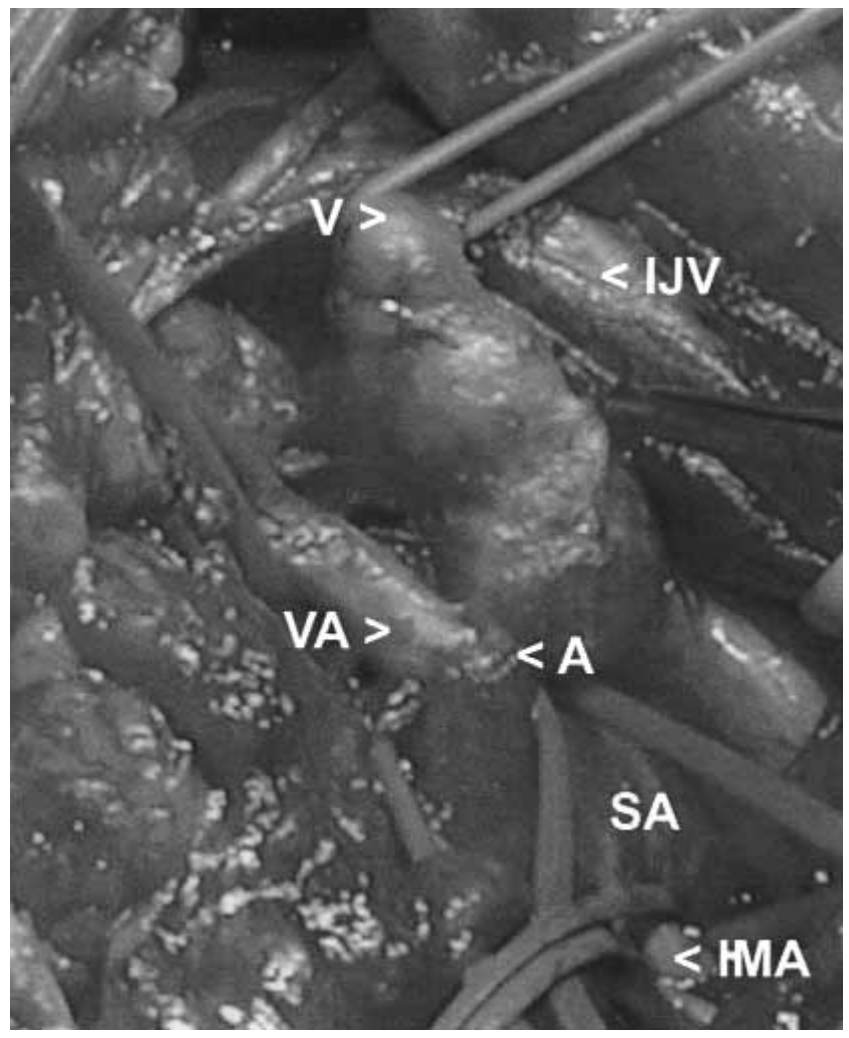

Figure 2. Operative photograph after isolation of the structures. IJV, Internal jugular vein; $S A$, subclavian artery; VA, vertebral artery; IMA, internal thoracic artery; $A$, arterial site of the AV communication; $V$, venous site of the AV communication.

fistula was ligated, the fistula was opened longitudinally, and the arterial origin was oversewn transversely with 6-0 polypropylene suture on the vertebral artery. The histologic specimen showed connective tissue consisting of a great amount of fibroblasts with patches of endothelial cover. The postoperative course was uneventful, and the locomotive noise heard by the patient disappeared. A duplex sonogram on the first follow-up visit, 4 weeks after the operation, showed normal anatomy and flow.

\section{Discussion}

AV communications in the neck result from trauma or from a medical intervention, most frequently a central venous puncture for hemodynamic monitoring or for parenteral nutrition. ${ }^{1}$ In this case, the complication developed after internal jugular vein puncture for mitral valve replacement. Arterial puncture was not noticed during insertion and central venous pressure was not elevated during mitral valve replacement surgery. There were no signs or symptoms raising the suspicion of AV fistula. The first symptoms were a noise and mild dyspnea experienced by the patient, both of which disappeared after AV fistula resection. The exact mechanism for creation of the AV communication in this case is unknown. It can be speculated that the guide wire perforated both the internal jugular vein and the vertebral artery; otherwise, arterial puncture would 
have been noticed. Most AV communications in the neck after central venous puncture develop between the vertebral artery and jugular vein. In the operative photograph, the two involved structures are much closer to each other than expected, and the usual direction of the needle during puncture is toward the vertebral artery. In agreement with others, this case demonstrates that AV communication can develop without any alarming sign of an arterial puncture. ${ }^{2} \mathrm{AV}$ communications, even those with no or mild symptoms, should be treated either surgically or by endovascular therapy, ${ }^{3}$ avoiding further complications such as infection, thrombosis, and arterio-arterial embolization. ${ }^{2}$

Endovascular therapy is safe and-being less invasive-is the treatment of choice using embolic coils or detachable balloons, ${ }^{1,3}$ if these tools and the expertise of selective catheterization are readily available.

\section{References}

1. Burger T, Tautenhahn J, Grote R, Halloul Z. Diagnosis and management of trauma and iatrogenic induced arteriovenous fistulas in the neck. Vasa. 1999;28:297-300.

2. Vinchon M, Laurian C, George B, D'Arigo G, Reizine D, Aymard A, et al. Vertebral arteriovenous fistulas: a study of 49 cases and review of the literature. Cardiovasc Surg. 1994;2:359-69.

3. Ricolfi F, Valiente E, Bodson F, Poquet E, Chiras J, Gaston A. Arteriovenous fistulae complicating central venous catheterization: value of endovascular treatment based on series of seven cases. Intensive Care Med. 1995;21:1043-7. 